\title{
MORBIMORTALIDADE EM IDOSOS POR FRATURA PROXIMAL DO FÊMUR
}

\author{
Gerardo Vasconcelos Mesquitaํ, Myrna Amélia Lobão Teixeira de Abreu Lima², Ana Maria Ribeiro dos \\ Santos $^{3}$, Eucário Leite Monteiro Alves ${ }^{4}$, José Nazareno Pearce de Oliveira Brito ${ }^{5}$, Maria do Carmo de \\ Carvalho e Martins ${ }^{6}$
}

\footnotetext{
${ }^{1}$ Doutor em Cirurgia Traumato-Ortopédica. Professor da Faculdade de Saúde, Ciências Humanas e Tecnológicas do Piauí NOVAFAPI. Professor Adjunto da Universidade Federal do Piauí (UFPI). Piauí. Brasil. E-mail: gvmesquita@uol.com.br

2 Fisioterapeuta. Piauí. Brasil. E-mail: myrna05amelia@yahoo.com.br

${ }^{3}$ Mestre em Enfermagem. Professora da Faculdade NOVAFAPI. Professora Assistente da UFPI. Piauí, Brasil. E-mail: ana. mrsantos@gmail.com

${ }^{4}$ Doutor em Cirurgia Torácica e Cardiovascular. Professor da Faculdade NOVAFAPI. Piauí, Brasil. E-mail: ealves@novafapi. com.br

${ }^{5}$ Doutor em Ciências Médicas. Professor da Faculdade NOVAFAPI. Piauí, Brasil. E-mail: nazapearce@uol.com.br

${ }^{6}$ Doutora em Ciências Biológicas. Professora da Faculdade NOVAFAPI. Professora da UFPI. Piauí, Brasil. E-mail: ccarvalho@ novafapi.com.br
}

\begin{abstract}
RESUMO: O aumento do número de idosos é uma realidade em nosso país, trazendo à tona a discussão sobre eventos incapacitantes nesta faixa etária, merecendo destaque a ocorrência de quedas, por suas conseqüências físicas, psicológicas e sociais. Uma conseqüência importante é a fratura proximal do fêmur, responsável por grande parte das cirurgias e internações, representando altos custos para a saúde pública. Realizou-se estudo bibliográfico que objetivou analisar a morbimortalidade por este tipo de fratura em publicações nas bases de dados MEDLINE, LILACS e SciELO, referentes ao período janeiro de 2003 a dezembro de 2007. A taxa média de mortalidade no primeiro ano pós-trauma foi de 21,8\%. A idade avançada, doenças prévias associadas e o sexo masculino são os fatores de maior contribuição para a ocorrência de óbito entre os idosos.
\end{abstract}

DESCRITORES: Envelhecimento. Mortalidade. Morbidade. Ferimentos e lesões.

\section{MORBID-MORTALITY IN ELDERLY DUE TO PROXIMAL FRACTURES OF THE FEMUR}

\begin{abstract}
The increase in the number of elderly is a reality in Brazil, which raises the discussion about incapacitating events among this age group. The occurrence of falls among Brazilian elderly deserves special mention because of their physical, psychological, and social consequences. An important consequence is a proximal fracture of the femur, responsible for a large percentage of surgeries and hospital admissions, representing high costs to public health. A bibliographic study was carried out with the objective of analyzing the morbid-mortality of this type of fracture in publications listed in the MEDLINE, LILACS, and SciELO data bases from January of 2003 to December of 2007. The average rate of mortality in the first year after-trauma was $21,8 \%$. Advanced age, previous associated illnesses, and the male gender are the factors of greatest contribution to the occurrence of death from such occurrences among Brazilian elderly.

DESCRIPTORS: Aging. Mortality. Morbidity. Wounds and injuries.
\end{abstract}

\section{MORBIMORTALIDAD EN ANCIANOS POR FRACTURA DE FÉMUR PROXIMAL}

RESUMEN: El aumento del número de ancianos es una realidad en nuestro país, lo que ha producido un debate acerca de los hechos que producen discapacidad en esta franja etaria, destacándose la ocurrencia de caídas, por sus consecuencias físicas, psicológicas y sociales. Una consecuencia importante es la fractura de fémur proximal, responsable por gran parte de las cirugías e internaciones, representando altos costos para la salud pública. Se realizó un estudio bibliográfico cuyo objetivo fue analizar la morbimortalidad por ese tipo de fractura en publicaciones en las bases de datos MEDLINE, LILACS y SciELO, referentes al período que va de enero de 2003 a diciembre de 2007. La tasa media de mortalidad en el primer año posterior al trauma fue de $21,8 \%$. La edad avanzada, las enfermedades previas asociadas y el sexo masculino son los factores de mayor contribución para la ocurrencia de óbitos en los ancianos.

DESCRIPTORES: Envejecimiento. Mortalidad. Morbosidad. Heridas y traumatismos. 


\section{INTRODUÇÃO}

O envelhecimento é definido como um processo progressivo, gradual e variável, caracterizado pela perda crescente de reserva funcional. Em conseqüência ocorrem alterações morfológicas, fisiológicas, bioquímicas e psicológicas, tornando o indivíduo mais propenso a adoecer, o que aumenta suas chances de morte. ${ }^{1-2}$ Essas mudanças naturais que ocorrem no envelhecimento, podem levar a limitações funcionais associadas a afecções agudas ou crônicas, tornando os idosos mais suscetíveis aos riscos ambientais, e conseqüentemente a ocorrência de quedas. ${ }^{3}$ Assim, devido ao crescente aumento da população idosa brasileira, muito se deve discutir sobre tais eventos incapacitantes nessa faixa etária. ${ }^{4}$

Nesse sentido, as fraturas de colo de fêmur, em idosos, ocupam um papel de grande importância, tanto pela sua freqüência, quanto pela sua gravidade,${ }^{5}$ visto que levam ao aumento da dependência e da mortalidade de aproximadamente $50 \%$, em um ano. ${ }^{6}$

Este estudo objetivou analisar a morbimortalidade em idosos por fratura proximal do fêmur, identificando a morbidade e mortalidade nestes casos, a prevalência dessas fraturas em relação ao gênero, o tipo de fratura de maior ocorrência, e aspectos do tratamento cirúrgico como tempo de espera para cirurgia e tempo de internação.

\section{ENVELHECIMENTO, ALTERAÇÕES DE SAÚDE E NECESSIDADES DE CUI- DADO}

O envelhecimento populacional caracterizase pela redução da participação relativa de crianças e jovens, acompanhada do aumento proporcional dos adultos e, particularmente dos idosos em uma sociedade. Em 2000, as crianças de zero a 14 anos correspondiam a $30 \%$ da população total e o contingente com 65 anos ou mais representava $5 \%$. Em 2050, ambos os grupos etários terão participação em torno de $18 \%$. $^{7}$

Este envelhecimento é um processo dinâmico, no qual há modificações morfológicas, funcionais, bioquímicas e psicológicas levando à perda da capacidade de adaptação do indivíduo ao meio ambiente, o que pode ocasionar maior vulnerabilidade e maior incidência de processos patológicos que terminem por levar à morte. ${ }^{8}$ Muitas das alterações fisiológicas associadas ao envelhecimento resultam de uma perda gradual que pode ter início na vida adulta, porém, devido à composição redundante dos sistemas orgânicos, essas perdas só se tornam importantes quando o declínio é extenso. ${ }^{9}$

Dentre as alterações anatômicas que ocorrem com o envelhecimento podemos citar as modificações na composição e forma do corpo. O envelhecimento leva a perda em estatura da ordem de $1 \mathrm{~cm}$ por década, aproximadamente, que começa a acontecer por volta dos 40 anos de idade. ${ }^{1-2}$ Outras condições que contribuem para a perda do tecido ósseo, seriam a redução de vitamina $\mathrm{D}$ pela pele e diminuição da absorção intestinal de cálcio. ${ }^{10}$

\section{Queda como fator causal da fratura proximal do fêmur}

No decorrer do ciclo da vida podem acontecer situações que resultem em perda de autonomia e independência, sendo uma delas, a queda. Quando relacionadas ao idoso são consideradas de caráter relevante, pois podem levá-los à incapacidade, injúria e morte. O custo social é proporcional à diminuição da autonomia e da independência ou a necessidade de institucionalização. ${ }^{11}$

A queda é o acidente que ocorre com maior freqüência no idoso, sendo suas complicações a principal causa de morte naqueles com mais de 65 anos. ${ }^{5} \mathrm{~A}$ mortalidade anual em idosos devido às quedas apresenta um pico por volta dos 85 anos e, somente cerca da metade dos idosos admitidos em um hospital após esse acidente, permanece viva no ano seguinte. ${ }^{12}$

A etiologia da queda é multifatorial, resultando da interação entre fatores intrínsecos e extrínsecos, sendo que a maior parte das quedas entre os idosos, tanto institucionalizados quanto na comunidade ocorre durante as atividades diárias. ${ }^{12-13}$

Os fatores de risco intrínsecos se relacionam ao próprio paciente e refletem incapacidade, pelo menos parcial, de manter ou de recuperar o equilíbrio quando houver um deslocamento acentuado do centro de gravidade localizado na região sacral. Tais fatores se referem à redução funcional em um ou mais sistemas sensório-motores essenciais ao controle da postura, às doenças agudas ou crônicas, aos transtornos cognitivos e comportamentais, à fraqueza da musculatura dos membros inferiores e o uso de medicamentos. ${ }^{13}$

Os fatores extrínsecos estão relacionados ao ambiente. Mais de $70 \%$ das quedas ocorrem em casa, e o risco se torna maior se o idoso vive só. Fa- 
tores ambientais podem ter um papel importante em até metade de todas as quedas. Portanto, tornase importante averiguar aspectos relacionados à: iluminação inadequada, superfícies escorregadias, tapetes soltos ou com dobras, degraus altos ou estreitos, obstáculos no caminho (móveis baixos, pequenos objetos, fios), ausência de corrimãos em corredores e banheiros, prateleiras excessivamente baixas ou elevadas, calçados inadequados e/ou patologias dos pés, maus-tratos e roupas excessivamente compridas. ${ }^{14}$

\section{Fisioterapia: prevenção de quedas e reabili- tação pós-cirúrgica}

A fisioterapia é o campo de atuação profissional cujo objeto de trabalho é o movimento humano e, através dele, intervém em todos os níveis em que possa se expressar, seja, prevenindo distúrbios que afetem o movimento, bem como recuperando um prejuízo sobre uma função ou mesmo adaptando o movimento prejudicado. ${ }^{15}$

Dentre as doenças osteoarticulares, as mais prevalentes no idoso, e que freqüentemente causam desequilíbrio e incapacidades, são: osteoporose, osteoartrite e artrite reumatóide. O tratamento fisioterapêutico direciona-se à manutenção do equilíbrio como forma de normalizar a vida do idoso afastando os fatores de risco comuns na terceira idade. As intervenções dependem do quadro apresentado durante a avaliação físico-funcional sendo de fundamental importância, a adesão do paciente, família e/ou cuidadores em todo o processo. ${ }^{16}$

Dessa forma, a prescrição de exercícios físicos para prevenção de quedas, manutenção ou ganho de equilíbrio no idoso é importante para obtenção de fortalecimento da musculatura abdominal e lombar, principalmente, além da musculatura dos membros inferiores. ${ }^{16}$

\section{METODOLOGIA}

Realizou-se levantamento bibliográfico utilizando os descritores aging (envelhecimento), mortality (mortalidade), morbidity (morbidade), wounds (ferimentos) e injuries (lesões), nos bancos de dados MEDLINE, LILACS e SciELO, referente ao período de janeiro de 2003 a dezembro de 2007. Como critério de inclusão, considerou-se artigos cuja população de idosos estudada apresentava fratura proximal de fêmur, tratamento cirúrgico com fixação interna ou artroplastia e história de primeira hospitalização para correção cirúrgica de fratura proximal de fêmur e, como critérios de exclusão artigos cuja população de idosos apresentava outras fraturas, fraturas de fêmur de natureza neoplásica e alterações degenerativas do quadril. Foram localizados 25 artigos, dos quais foram selecionados 14 de acesso aberto relacionado com a temática. Efetuou-se então análise estatística descritiva sendo as variáveis organizadas através de freqüência absoluta e percentual, considerando a média das taxas de mortalidade, causadas por fratura de fêmur em idosos, de acordo com a taxa de mortalidade pós-trauma, índice de fratura por tipo e distribuição por sexo, encontrada em cada artigo.

\section{RESULTADOS E DISCUSSÃO}

O número de idosos com Fratura Proximal de Fêmur (FPF) envolvido nos estudos levantados, correspondeu a 4.013. A fratura proximal de fêmur descrita nas publicações foi classificada por tipo, como - Colo do Fêmur (CF), Transtrocanteriana (TT), Subtrocanteriana (ST). Dentre os artigos selecionados, nove apresentam caráter prospectivo, cinco retrospectivo, com maior concentração nos anos de 2006 e 2007 e 11 apresentam taxa de mortalidade anual.

Constata-se na Tabela 1 que a taxa de mortalidade no primeiro ano pós-trauma alcançou um percentual de $21,8 \%$, a ocorrência de FPF em homens atingiu $27,4 \%$, enquanto que nas mulheres a taxa foi de $72,5 \%$. As fraturas foram classificadas por tipo, como: fratura do CF, TT e ST. Pôde-se observar uma diferença percentual pequena entre as TT ( $50 \%)$ e as CF ( $45 \%)$.

Observa-se que os pacientes idosos com FPF apresentam altos índices de mortalidade por fratura de colo de fêmur. Pesquisas apontam que a taxa de mortalidade varia de $20 \%$ a $30 \%$ no primeiro ano após a intervenção cirúrgica. ${ }^{30}$ Nos artigos levantados, os índices variaram de $7,4 \%$ a $34,8 \%$, com uma taxa média de mortalidade de $21,8 \%$.

Os homens idosos apresentam maiores taxas de óbito pós FPF, ${ }^{23-24,26}$ entretanto existe estudo que não associa a população masculina com esse índice de óbito, ${ }^{31}$ verificando-se assim uma controvérsia quanto à prevalência do sexo para mortalidade após FPF. 
Tabela 1 - Taxa de mortalidade anual pós- trauma, distribuição por sexo e tipo de fratura de 2003 a 2007

\begin{tabular}{|c|c|c|c|c|c|c|c|c|c|c|c|c|c|}
\hline \multirow{3}{*}{$\begin{array}{l}\text { Artigo } \\
\text { Autor/ano }\end{array}$} & & \multirow{2}{*}{\multicolumn{2}{|c|}{$\begin{array}{c}\text { Taxa de } \\
\text { Mortalidade }\end{array}$}} & \multicolumn{4}{|c|}{$\begin{array}{l}\text { Índice de fratura por } \\
\text { sexo }(n=2300)\end{array}$} & \multicolumn{5}{|c|}{$\begin{array}{l}\text { Índice por tipo de fratura } \\
(n=1381)\end{array}$} & \multirow{3}{*}{$\begin{array}{c}\text { Total } \\
\text { pacientes }\end{array}$} \\
\hline & & & & \multicolumn{2}{|c|}{ Masculino } & \multicolumn{2}{|c|}{ Feminino } & \multicolumn{2}{|r|}{$\mathrm{CF}$} & \multicolumn{2}{|c|}{ TT } & ST & \\
\hline & & $f$ & $\%$ & f & $\%$ & $f$ & $\%$ & $f$ & $\%$ & $f$ & $\%$ & & \\
\hline Canto et al. ${ }^{17}$ & 2003 & - & - & - & - & - & - & 42 & 50,0 & 42 & 50,0 & $-\quad-$ & 84 \\
\hline Roder et al. ${ }^{18}$ & 2003 & 17 & 11,7 & 29 & 20,0 & 116 & 80,0 & - & - & - & - & $-\quad-$ & 145 \\
\hline Nurmi et al. ${ }^{19}$ & 2004 & 34 & 32,0 & 32 & 30,2 & 74 & 69,8 & 65 & 61,3 & 35 & 33,0 & 65,6 & 106 \\
\hline Silveira et al. ${ }^{20}$ & 2005 & - & - & 95 & 24,9 & 287 & 75,1 & - & - & - & - & $-\quad-$ & 382 \\
\hline Cunha; Veado ${ }^{21}$ & 2006 & 38 & 25,0 & - & - & - & - & 67 & 44,0 & 77 & 50,0 & $-\quad-$ & 153 \\
\hline Pinheiro et al. ${ }^{22}$ & 2006 & 130 & 21,5 & 186 & 30,7 & 420 & 69,3 & - & - & - & - & $-\quad-$ & 606 \\
\hline Garcia et al. ${ }^{23}$ & 2006 & 17 & 30,3 & 47 & 84,0 & 9 & 16,0 & 21 & 38,0 & 25 & 45,0 & 57,3 & 56 \\
\hline \multirow[t]{2}{*}{ Fierens; Broos ${ }^{24}$} & 2006 & $92^{*}$ & 24,0 & - & - & - & - & - & - & - & - & $-\quad-$ & 384 \\
\hline & & $253 \dagger$ & 23,0 & - & - & - & - & - & - & - & - & $-\quad-$ & 1102 \\
\hline Laranjeira et al..$^{25}$ & 2007 & 19 & 18,4 & 36 & 35,3 & 66 & 64,7 & 51 & 50,0 & 49 & 48,0 & 22,0 & 102 \\
\hline Souza et al. ${ }^{26}$ & 2007 & 29 & 7,4 & 114 & 29,2 & 286 & 70,8 & 149 & 38,2 & 209 & 53,6 & 307,7 & 390 \\
\hline Muniz et al. ${ }^{27}$ & 2007 & 13 & 14,6 & 34 & 38,2 & 55 & 61,8 & 34 & 38,2 & 52 & 58,4 & 22,2 & 89 \\
\hline Dzupa et al. ${ }^{28}$ & 2007 & 85 & 34,8 & 58 & 24,0 & 186 & 76,0 & 115 & 47,0 & 117 & 47,0 & 166,0 & 244 \\
\hline Haentjens et al. ${ }^{29}$ & 2007 & 32 & 18,8 & - & - & 170 & 100,0 & 84 & 49,0 & 86 & 51,0 & $-\quad-$ & 170 \\
\hline Total & & 759 & 21,8 & 631 & 27,4 & 1669 & 72,6 & 628 & 45,5 & 692 & 50,1 & 614,4 & 4013 \\
\hline
\end{tabular}

CF: fratura de colo de fêmur, TT: fratura transtrocanteriana, ST: fratura subtrocanteriana

* considerado o período de 1978 a 1983, † considerado o período de 1998 a 2003

Alguns autores reportam a associação da idade com a mortalidade após FPF. Pacientes com idade acima de 80 anos apresentam maior probabilidade de morte após esse tipo de fratura se comparados com indivíduos na faixa etária de 60 a 80 anos. ${ }^{20,23} \mathrm{~A}$ taxa de incidência de FPF em idosos com idade acima de 80 anos é aproximadamente dez vezes maior que naqueles com idade acima de 45 anos e quase 4 vezes maior que em pacientes na faixa etária de 70 a 79 anos..$^{20,31}$

Quanto ao índice de fratura por sexo observa-se diversidade de opiniões. Nesse sentido, alguns estudos mostram que a incidência de FPF predominou no sexo feminino, ${ }^{18-20,25-29,31}$ porém em outro estudo, foi encontrada uma diferença percentual de $68 \%$ a mais para o sexo masculino. ${ }^{23}$ Entretanto, há relato de que a partir dos 50 anos, a freqüência desse tipo de fratura é duas vezes mais elevada no sexo feminino em relação ao masculino. Registrando-se um percentual de 15 a $20 \%$ de morte dentro do primeiro ano após a fratura e cerca de $50 \%$ dos sobreviventes passando a necessitar de cuidados especiais a longo prazo. ${ }^{32}$
A predominância da mulher também foi relatada em estudos que analisaram idosos com fratura do CF após artroplastia parcial do quadril ${ }^{33}$ e em estudos epidemiológicos retrospectivos. ${ }^{20,34}$ Esses últimos assinalam que esta maior freqüência pode ser explicada pelo fato do sexo feminino ser mais exposto aos fatores de risco, pela maior prevalência de osteoporose, maior suscetibilidade para quedas, maior expectativa de vida do que os homens, além das influências antropométricas e fatores genéticos relacionados ao sexo.

A correlação entre o tipo de fratura com a faixa etária foi relatada em estudo epidemiológico sobre FPF. As fraturas de CF ocorreram predominantemente entre a sétima e oitava décadas e as TT entre a oitava e nona décadas, sendo que as ST ocorrem isoladamente em menor número na terceira e nona décadas. ${ }^{34}$

De acordo com a Tabela 2, os pacientes tinham em média 6,8 dias de espera para realização da intervenção cirúrgica desde a data da fratura, passavam em média 13 dias internados e a maioria deles $(88,0 \%)$ apresentava doenças prévias asso- 
ciadas. Em dois artigos foi encontrada referência quanto ao número de doenças prévias associadas por paciente, sendo em média 2,6.

Assim sendo, outro ponto a considerar se refere às comorbidades, as quais têm sido utilizadas como precursoras da mortalidade após fratura proximal do fêmur. O efeito das comorbidades sobre a mortalidade tem sido medido tanto pela quantidade de doenças coexistentes, quanto pelo seu tipo. Pacientes com maior número de doenças coexistentes têm maior possibilidade de morrer. ${ }^{26}$
Em relação a doenças prévias existentes nos pacientes idosos, relatadas à época da fratura, as de maior influência foram: doenças cardíacas, pulmonares, renais, acidente vascular cerebral e Diabetes mellitus. ${ }^{26}$

Estudo que analisou 30 pacientes idosos com idade entre 70 a 95 anos, refere que a coexistência de uma doença a mais aumenta a chance de óbito em aproximadamente $44,0 \%{ }^{26}$ Entretanto, neste levantamento bibliográfico, os resultados encontrados entre a associação da mortalidade por FPF com o número de comorbidades não foram conclusivos pois apenas dois estudos fizeram referência a tal fato.

Tabela 2 - Pacientes com doenças prévias, média de tempo de espera para cirurgia e internação, média de doenças prévias de 2003 a 2007

\begin{tabular}{lccccc}
\hline $\begin{array}{l}\text { Artigos } \\
\text { Autor/ano }\end{array}$ & \multicolumn{2}{c}{$\begin{array}{c}\text { Pacientes com doenças prévias } \\
\%\end{array}$} & MTCir & MTlnt & MDPrévias \\
\hline Canto et al. ${ }^{17}$ & 2003 & - & - & - & - \\
Roder et al. ${ }^{18}$ & 2003 & - & - & - & - \\
Nurmi et al. ${ }^{19}$ & 2004 & - & 1,5 dias & 8,5 dias & - \\
Silveira et al. ${ }^{20}$ & 2005 & - & - & - & - \\
Cunha; Veado. ${ }^{21}$ & 2006 & - & 4,1 dias & - & - \\
Pinheiro et al. ${ }^{22}$ & 2006 & - & - & - & - \\
Garcia et al. ${ }^{23}$ & 2006 & 100,0 & 6,8 dias & - & 3,3 \\
Fierens; Broos ${ }^{24}$ & 2006 & - & - & - & - \\
Pinheiro et al. ${ }^{31}$ & 2006 & - & - & - & - \\
Laranjeira et al. ${ }^{25}$ & 2007 & 81,4 & 14,8 dias & 20,5 dias & 1,9 \\
Souza et al. ${ }^{26}$ & 2007 & 82,8 & - & - & - \\
Muniz et al. ${ }^{27}$ & 2007 & - & - & - & - \\
Dzupa et al. ${ }^{28}$ & 2007 & - & - & - & - \\
Haentjens et al. ${ }^{29}$ & 2007 & - & 6,8 dias & 13,06 dias & 2,6 \\
\hline Média & & 88,0 & dias & - \\
\hline
\end{tabular}

MTCir: média de tempo de espera para cirurgia desde a fratura, MTInt: média de tempo de internação MDPrévia: quantidade média de doenças prévias

Verificou-se que o tempo médio de espera entre a fratura e a cirurgia foi 6,8 dias. Esse tempo de espera para a cirurgia se justifica em função da necessidade de se estabilizar clinicamente o paciente de maior gravidade e o acréscimo de um dia no tempo de espera para a realização da cirurgia aumenta a possibilidade de óbito em cerca de $4 \% .{ }^{26}$ No entanto, em alguns estudos levantados, não ficaram claros os motivos que levaram o tempo de espera pelo procedimento cirúrgico.

Observou-se grande diferença entre os dias de permanência hospitalar nos artigos pesquisados, variando de 4,1 a 20,5 dias, com uma média de 13 dias. No entanto, os autores não esclarecem quais fatores influenciaram nessa permanência hospitalar, valendo ressaltar que o aumento no período de internação é um fator agravante para complicações e pode influenciar na taxa de morbimortalidade.

\section{CONCLUSÃO}

A população idosa encontra-se em constante crescimento nos últimos anos em nosso país, observando-se que as quedas, associadas à FPF, 
são secundárias ao processo de envelhecimento. Constatou-se que as comorbidades associadas, presentes nesta etapa da vida e apresentadas por $88 \%$ dos pacientes, com uma média de 2,6 doenças prévias associadas, contribuem para a mortalidade por FPF, no decorrer do primeiro ano de $21,8 \%$. Verificou-se também que este tipo de fratura apresenta incidência maior no sexo feminino, correspondendo a $72,5 \%$ e que as fraturas do CF e TT são as que mais ocorrem, aproximadamente numa mesma proporção. Identificou-se ainda que os pacientes tiveram tempo médio de permanência hospitalar de 13 dias, sendo a média de tempo para cirurgia de 6,8 dias. Dessa forma, as políticas de saúde objetivando programar medidas preventivas para diminuir os riscos de quedas na população idosa devem ser implementadas para melhorar a qualidade de vida, assim como reduzir o impacto socioeconômico que esses episódios acarretam.

\section{REFERÊNCIAS}

1. Porto CC, editor. Semiologia médica. $4^{\mathrm{a}}$ ed. Rio de Janeiro (RJ): Guanabara Koogan; 2001.

2. Rebelatto JR, Morelli JGS, editores. Fisioterapia geriátrica. A prática de assistência ao idoso. $1^{\mathrm{a}} \mathrm{ed}$. São Paulo (SP): Manole; 2004.

3. BritoFC, CostaSMN.Quedas. In: Papaléo Neto M, Brito FC, editores. Urgências em geriatria: epidemiologia, fisiopatologia, quadro clinico. Controle terapêutico. São Paulo (SP): Atheneu; 2001. p 43-62.

4. Perracini MR, Ramos LR. Fatores associados a quedas em um corte de idosos residentes na comunidade. Rev Saúde Pública 2002 Dez; 36(6):709-16.

5. Maciel ACC, Guerra RO. Prevalência e fatores associados ao déficit de equilíbrio em idosos. R Bras Ci Mov. 2005; 13(1):37-44.

6. Barbosa MLJ, Nascimento EFA. Incidência de internações de idosos por motivo de queda em hospital geral em Taubaté. Rev Biociênc. 2001 JanJun; 7(1):35-42.

7. Instituto Brasileiro de Geografia e Estatística IBGE [página da internet]. [acesso em 2008 Mai. 20]. Disponível em: http:/ / www.ibge.gov.br

8. Freitas EV, Py L, Néri AL, Cançado FAX, Gorzone ML, Rocha SN. Tratado de geriatria e gerontologia. Rio de Janeiro (RJ): Editora Guanabara Koogan, 2002.

9. Papaléo Netto M, Carvalho Filho ET, Salles RFN, editores. Fisiologia do envelhecimento. Geriatria, fundamentos, clínica e terapêutica. $2^{\mathrm{a}}$ ed. São Paulo (SP): Atheneu; 2006.

10. Figueiredo CP, Fuller R. Envelhecimento do sistema osteoarticular. In: Magnoni D, Cukier C, Oliveira PA, editores. Nutrição na terceira idade. São Paulo (SP): Sarvier; 2005.
11. Fabrício SCC, Rodrigues RAP, Costa Junior ML. Causas e conseqüências de quedas de idosos atendidos em hospital público. Rev Saúde Pública. 2004 Fev; 38(1):93-9.

12. Gallo JJ, Busby-Whitehead J, Rabins Peter V, Silliman RA, Murphy JBR, editores. Assistência ao idoso. Aspectos clínicos do envelhecimento. $5^{\mathrm{a}}$ ed. Rio de Janeiro (RJ): Guanabara Koogan; 2001.

13. Guimarães RM, Cunha UGV, editores. Sinais e sintomas em geriatria. $2^{a}$ ed. São Paulo (SP): Atheneu; 2004.

14. Hargreaves LHH, editor. Geriatria. $1^{\mathrm{a}}$ ed. Brasília (DF): Seep; 2006.

15. Suzuki HS, editor. Conhecimentos essenciais para atender bem o paciente idoso. São José dos Campos (SP): Pulso; 2003.

16. Montenegro SMR, Silva CAB. Os efeitos de um programa de fisioterapia como promotor de saúde na capacidade funcional de mulheres idosas institucionalizadas. Rev Bras Geriatr Gerontol. 2007 Ago; 10(2):161-78.

17. Canto RST, Silveira MA, Rosa AS, Gomide LC, Baraúna MA. Morfologia radiográfica de quadril e pelve e sua relação com fraturas proximais do fêmur. Rev Bras Ort. 2003 Jan-Fev; 38(2):12-20.

18. Roder F, Schwab M, Aleker T, Morike K, Thon KP, Klotz U. Proximal femur facture in older patientsreabilitation and clinical outcome. Age Ageing. 2003; 32(1):74-80.

19. Nurmi I, Narinen A, Luthje P, Tanninen S. Functional outcome and survival after hip fracture in elderly: a prospective study of 106 consecutive patients. J Orthopaed Traumatol. 2004; 4(1):7-14.

20. Silveira VAL, Medeiros MMC, Coelho-Filho J, Mota RS, Noleto JCS, Costa FS, et al. Incidência de fratura do quadril em área urbana do Nordeste brasileiro. Cad Saúde Pública. 2005 Mai-Jun; 21(3):907-12.

21. Cunha U, Veado MAC. Fratura de extremidade proximal do fêmur em idosos: independência funcional e mortalidade em um ano. Rev Bras Ort. 2006 Jun; 41(6):195-9.

22. Pinheiro RS, Coeli CM, Vidal EIO, Camargo Jr KR. Mortalidade após fratura proximal do fêmur. Cad Saude Col. 2006 Abr-Jun.; 14(2):327-36.

23. Garcia R, Leme MD, Leme LEG. Evolution of brazilian elderly with hip fracture secondary to a fall. Clinics. 2006; 61(6):539-44.

24. Fierens J, Broos PLO. Quality of life after hip fracture surgery in the elderly. Acta Chir Bel. 2006; 106(1):393-6.

25. Laranjeiras JA, Ribeiro TA, Guterres LW, Nascimento DZ, Fortes BC, Guerra V A. Estudo prospectivo da mortalidade e morbidade em fraturas do fêmur proximal em pacientes com 65 anos de idade ou mais. Anais XV Congresso Sul Brasileiro de Ortopedia Traumatologia 
- SULBRA; 2007 Jun 21-23; Gramado, Brasil. Gramado (RS): SOTRS; 2007.

26. Souza RC, Pinheiro RS, Coeli CM, Junior KRC, Torres TZG. Aplicação de medidas de ajuste de risco para a mortalidade após fraturar proximal de fêmur. Rev Saúde Pública. 2007 Ago; 41(4):625-31.

27. Muniz CF, Arnaute AC, Yoshida M, Trelha CS. Caracterização dos idosos com fatura de fêmur próximal atendidos em hospital escola público. Rev Esp Saúde. 2007 Jun; 8(2):33-8.

28. Dzupa V, Bartonicek J, Skála-Rosenbaum J, Prikazsky V. Mortality in patients with proximal femoral fractures during the first year after the injury. Bone. 2007; 41(6):958-64.

29. P. Haentjens, P. Autier, M. Barette, K. Venken, D. Vanderschueren, S. Boonen Haentjens Survival and functional outcome according to hip fracture type: a one-year prospective cohort study in elderly women with an intertrochanteric or femoral neck fracture. Bone. 2007; 41(6):958-64.

30. Hebert S, Xavier R, editores. Ortopedia e traumatologia. Princípios e prática. $3^{\text {a }}$ ed. Porto Alegre (RS): Artmed; 2003.

31. Pinheiro RS, Vieira RA, Coeli CM, Vidal EIO, Camargo Jr. KR. Utilização do SIH e do SIM para o cálculo de mortalidade hospitalar e em 30 dias para as internações de pacientes com fratura proximal de fêmur. Cad Saude Colet. 2006 Abr-Jun; 14(2):337-44.

32. Gulhan EG. Osteoporose e fraturas osteoporóticas. In: Pickles B, Compton A, Cott C, Simpson J, Vandervoort A, editores. Fisioterapia na Terceira idade. $2^{\mathrm{a}}$ ed. São Paulo (SP): Santos; 2000.

33. Chikude T, Fujiki EN, Honda EK, Ono NK, Milani C. Avaliação da qualidade de vida dos pacientes idosos com fratura do colo do fêmur tratados cirurgicamente pelo artroplastia parcial do quadril. Acta Ortop Bras. 2007; 15(4):191-6.

34. Rocha MA, Carvalho WS, Zanqueta C, Lemos S. Estudo epidemiológico retrospectivo das fraturas do fêmur proximal tratados no Hospital Escola da Faculdade de Medicina do Triângulo Mineiro. Rev Bras Ortop. 2001 Ago; 36(8):311-6. 